\title{
Preoperative Glasgow prognostic score as a predictor of primary bladder cancer recurrence
}

\author{
OZGUR HAKI YUKSEL ${ }^{1}$, SERKAN AKAN ${ }^{1}$, AHMET URKMEZ ${ }^{2}$, \\ CAGLAR YILDIRIM $^{1}$, AYTAC SAHIN ${ }^{1}$ and AYHAN VERIT ${ }^{1}$ \\ ${ }^{1}$ Department of Urology, Fatih Sultan Mehmet Research and Training Hospital, TR-34752 Istanbul; \\ ${ }^{2}$ Department of Urology, Haydarpasa Numune Research and Training Hospital, TR-34668 Istanbul, Turkey
}

Received February 12, 2016; Accepted April 27, 2016

DOI: $10.3892 / \mathrm{mco} .2016 .901$

\begin{abstract}
The correlation between systemic inflammatory markers and malignancies has been assessed by a number of recent studies. The aim of this study was to prospectively assess preoperative inflammation markers and Glasgow prognostic scores (GPS) in patients who underwent surgery for primary bladder cancer (BC), and evaluate the predictive value of GPS for disease recurrence and progression. A total of 38 patients (mean age, $60.16 \pm 9.71$ years; range, 33-76 years) who were treated in our department between May, 2014 and August, 2015 were enrolled in the present study. Preoperatively, patient information regarding gender, body mass index, serum C-reactive protein (CRP) and albumin levels, GPS and comorbidities, were collected and recorded. Transurethral resection of the bladder was performed, followed by histopathological evaluation of the resected material. The tumor size, stage and grade and the presence of necrosis were determined. According to the international TNM classification, the results of the histopathological analysis were as follows: Ta low$(n=24)$ and high-grade $(n=4)$; and T1 low- $(n=2)$ and high-grade $(\mathrm{n}=8)$. The median follow-up period was 10.1 months (range, 6-12 months). During this period, recurrence was observed in 10 cases and disease progression was detected in 1 patient. Hypoalbuminemia was encountered in $40 \%$ of the cases with recurrence, which was significantly higher compared with that in patients without recurrence $(7.1 \% ; \mathrm{P}=0.031)$. In patients who had recurrence, a GPS of 1-2 points and tumor necrosis were more frequently detected compared with those without recurrence (60 vs. $7.1 \%, \mathrm{P}=0.002$; and 80 vs. $7.1 \%, \mathrm{P}=0.001$, respectively). Excluding a cystectomized case with a diagnosis of muscle-invasive $\mathrm{BC}$, disease progression was not detected in any of the cases with recurrence during follow-up. Therefore,
\end{abstract}

Correspondence to: Dr Ahmet Urkmez, Department of Urology, Haydarpasa Numune Research and Training Hospital, 23 Tibbiye Street, Uskudar, TR-34668 Istanbul, Turkey

E-mail: ahmeturkmez@hotmail.com

Key words: bladder cancer, Glasgow prognostic score, inflammation, hypoalbuminemia we consider that GPS and serum markers of systemic inflammatory response may be used as predictors of recurrence in patients with transitional cell $\mathrm{BC}$.

\section{Introduction}

Approximately 54.000 novel cases of bladder cancer (BC) are diagnosed in the USA annually, with BC being the fourth most frequent malignancy in men and the eigth most common cause of cancer-related mortality (1). Transitional cell carcinoma (TCC) constitutes $90 \%$ of all BCs. The majority of newly diagnosed BCs $(75 \%)$ are non-muscle-invasive urothelial carcinomas, while invasion of the muscularis propria is detected in the remainder of cases $(25 \%)$. In the determination of prognosis, the most important factors are grade and stage of the tumor. Cystoscopy and tissue biopsy/resection are the standard diagnostic methods. To date, numerous tumor markers have been introduced for routine clinical use (2). Management of $\mathrm{BC}$ is important in order to monitor and reduce tumor recurrence and progression.

The identification of patients at higher risk of disease progression has been a focus of attention for several years. Improving our knowledge on the effects of different factors or their combination on the risk of progression and cancer-specific survival may prove beneficial. At present, although TNM stage and grade are the most widely used means of survival prediction, additional methods should be investigated.

Pathophysiological studies have demonstrated that, apart from the tumor characteristics, the immune response developed by the host affects disease progression. Accumulating evidence suggests an important role of local and systemic inflammatory responses in the progression of various solid tumors (3).

The predictive value of the systemic inflammatory response (SIR) for cancer-specific survival has not been fully elucidated. However, as components of the SIR, it is known that pro-inflammatory cytokines and growth factors are released into the systemic circulation. Furthermore, tumor growth factors have been considered to increase the release of cytokines. SIR may be activated secondary to local tissue damage caused by interactions of tumor necrosis factors or tumor host cells (4).

The present study prospectively examined the data of patients who underwent surgery for primary $\mathrm{BC}$, regarding 
preoperative inflammation markers and Glasgow prognostic scores (GPS), and evaluated their impact in the prediction of disease recurrence and progression.

\section{Patients and methods}

Patient selection and data collection. This study was performed on a total of 38 patients with urothelial BC who were hospitalized between May, 2014 and August, 2015. The patients were graded and staged according to the TNM classification of the World Health Organization criteria of urological pathology as follows: Ta low-grade (LG) $(n=24)$, Ta high-grade $(\mathrm{HG})(\mathrm{n}=4)$, T1 LG $(n=2)$ and T1 HG $(n=8)$. Preoperatively, patient information regarding age, gender, body mass index (BMI) and comorbidities [diabetes mellitus (DM), hypertension (HT) and coronary artery diseases (CAD)] was collected and recorded.

Laboratory measurements. Preoperatively, routine laboratory measurements, whole-blood counts, C-reactive protein (CRP) and albumin levels were recorded. GPSs were evaluated using SIR markers. Patients with elevated CRP levels $(>1 \mathrm{mg} / \mathrm{dl})$ in combination with hypoalbuminemia $(<3.5 \mathrm{~g} / \mathrm{dl})$ were allocated 2 points. Patients with elevated CRP alone or hypoalbuminemia alone were allocated 1 point, while patients within normal limits were allocated 0 points (Table I).

Pathological analysis. All the surgical specimens were examined by an experienced genitourinary pathologist and processed according to standardized procedures. TNM stage was determined according to the International Union for Cancer Control/American Joint Cancer Committee 2004 classification and tumor grade was determined according to the World Health Organization 1973 and 2004 classifications (htt ps://uroweb.org/guideline/non-muscle-invasive-bladder-cancer/). In addition, the presence of tumor necrosis was recorded.

Statistical analysis. Values are expressed as the mean \pm standard deviation or as the frequency as appropriate. Statistical analysis was performed using SPSS version 22 (IBM SPSS, Istanbul, Turkey). Parameters with normal distribution were evaluated using the Kolmogorov-Smirnov test, and fitness of data to normality was determined. Comparison of quantitative data and intergroup comparisons of parameters were performed using the Student's t-test. For the comparison of qualitative data, the Fisher's exact $\chi^{2}$ test was used and risk calculations were performed. $\mathrm{P}<0.05$ was considered to indicate a statistically significant difference between values.

Ethical approval and informed consent. The protocol of the present study was reviewed and approved by the Regional Ethical Vetting Board (İstanbul, Turkey), and was in accordance with the ethical standards laid down by the 1964 Declaration of Helsinki and its later amendments. Informed consent was obtained from all individual participants included in the study.

\section{Results}

Patient characteristics. A total of 38 patients, namely 4 women (10.5\%) and 34 men $(89.5 \%)$, with a mean age of $60.16 \pm 9.71$ years (range, 33-76 years), who were diagnosed
Table I. Glasgow prognostic score.

\begin{tabular}{lc} 
Variables & Score \\
\hline $\mathrm{CRP} \leq 1 \mathrm{mg} / \mathrm{dl}$, albumin $\geq 3.5 \mathrm{~g} / \mathrm{dl}$ & 0 \\
$\mathrm{CRP}>1 \mathrm{mg} / \mathrm{dl}$, albumin $\geq 3.5 \mathrm{~g} / \mathrm{dl}$ & 1 \\
$\mathrm{CRP} \leq 1 \mathrm{mg} / \mathrm{dl}$, albumin $<3.5 \mathrm{~g} / \mathrm{dl}$ & 1 \\
$\mathrm{CRP}>1 \mathrm{mg} / \mathrm{dl}$, albumin $<3.5 \mathrm{~g} / \mathrm{dl}$ & 2
\end{tabular}

CRP, C-reactive protein.

with muscle-invasive BC, were enrolled in the present study. The cases were categorized based on histopathological results as follows: Ta LG $(n=24)$, Ta HG $(n=4), T 1 \quad L G(n=2)$ and T1 HG ( $\mathrm{n}=8)$. The median follow-up period was 10.1 months (range, 6-12 months). The follow-up protocol consisted of control cystoscopies performed at 3-month intervals. A total of 10 patients who were staged as T1 based on histopathology results underwent repeat transurethral resection of the bladder (TUR-M) 1 month later. All the patients received a single dose of $40 \mathrm{mg}$ mitomycin as an intravesical instillation during the postoperative period, whereas 14 patients who were classified as T1 or HG were scheduled for Bacille Calmette-Guérin induction therapy. During the follow-up period, recurrence occurred in 10 patients and disease progression in 1 patient. The patients developing recurrence had T1 HG $(\mathrm{n}=6)$, Ta HG $(n=2)$ and Ta LG $(n=2)$ tumors. One patient who had a T1 HG tumor at baseline underwent repeat TUR-M 1 month later and histopathological analysis revealed tumor progression, which necessitated cystectomy, confirming the diagnosis of muscle-invasive BC. No deaths were reported during the follow-up period.

Tumor characteristics and laboratory parameters. As shown in Table II, the tumor diameter ranged between 6 and $70 \mathrm{~mm}$ (mean, $28.79 \pm 15.43 \mathrm{~mm}$ ). The CRP levels were $0.01-1.89 \mathrm{mg} / \mathrm{dl}$ (mean, $0.48 \pm 0.53 \mathrm{mg} / \mathrm{dl}$ ); elevated CRP was detected in 10 cases $(26.3 \%)$, while in 28 patients $(73.7 \%)$ the CRP levels were within the normal range $(0-0.5 \mathrm{mg} / \mathrm{dl})$. The albumin levels ranged between 3.30 and $4.70 \mathrm{~g} / \mathrm{dl}$ (mean, $4.13 \pm 0.45 \mathrm{~g} / \mathrm{dl}$ ), while the normal range was $3.5-5.2 \mathrm{~g} / \mathrm{dl}$; hypoalbuminemia was detected in 6 cases $(15.8 \%)$, while the serum albumin levels were normal in 32 cases $(84.2 \%)$. DM ( $n=6$; $15.8 \%)$, HT $(\mathrm{n}=14 ; 36.8 \%)$ and CAD $(\mathrm{n}=14 ; 36.8 \%)$ were also observed. GPS scores of $0(n=30 ; 78.9 \%), 1(n=6 ; 15.8 \%)$ and $2(\mathrm{n}=2 ; 5.3 \%)$ were estimated for the patient cohort. The tumor stage was $\mathrm{T} 1$ and $\mathrm{Ta}$ in $11(26.3 \%)$ and $28(73.7 \%)$ patients, respectively. LG and HG tumors were present in $26(68.4 \%)$ and $12(31.6 \%)$ patients, respectively. Tumor necrosis was observed in 10 cases $(26.3 \%$ ), while there was no sign of necrosis in 28 patients (73.7\%). Recurrence was observed in 10 of the 38 cases $(26.3 \%)$.

Association of clinicopathological parameters with the recurrence of primary $B C$. The association of tumor recurrence with various clinicopathological parameters is shown in Table III. No statistically significant difference was found between patients with and those without recurrence with regard to mean age, 
Table II. Distribution of study parameters.

A, mean values of baseline parameters according to recurrence and non-recurrence

\begin{tabular}{lcc}
\hline Parameters & Min-max & Mean \pm SD \\
\hline Tumor size $(\mathrm{mm})$ & $6-70$ & $28.79 \pm 15.43$ \\
CRP $(\mathrm{mg} / \mathrm{dl})$ & $0.01-1.89$ & $0.48 \pm 0.53$ \\
Albumin $(\mathrm{g} / \mathrm{dl})$ & $3.30-4.70$ & $4.13 \pm 0.45$ \\
\hline
\end{tabular}

$\mathrm{B}$, no. of patients according to recurrence and non- recurrence

\begin{tabular}{|c|c|c|}
\hline Parameters & $\mathrm{n}$ & $\%$ \\
\hline \multicolumn{3}{|l|}{$\mathrm{DM}$} \\
\hline Yes & 6 & 15.8 \\
\hline No & 32 & 84.2 \\
\hline \multicolumn{3}{|l|}{ HT } \\
\hline Yes & 14 & 36.8 \\
\hline No & 24 & 63.2 \\
\hline \multicolumn{3}{|l|}{ CAD } \\
\hline Yes & 14 & 36.8 \\
\hline No & 24 & 63.2 \\
\hline \multicolumn{3}{|c|}{ Elevated CRP } \\
\hline Yes & 10 & 26.3 \\
\hline No & 28 & 73.7 \\
\hline \multicolumn{3}{|c|}{ Hypoalbuminemia $(<3.5 \mathrm{~g} / \mathrm{dl})$} \\
\hline Yes & 6 & 15.8 \\
\hline No & 32 & 84.2 \\
\hline \multicolumn{3}{|l|}{ GPS } \\
\hline 0 & 30 & 78.9 \\
\hline 1 & 6 & 15.8 \\
\hline 2 & 2 & 5.3 \\
\hline \multicolumn{3}{|l|}{ Tumor stage } \\
\hline $\mathrm{T} 1$ & 10 & 26.3 \\
\hline $\mathrm{Ta}$ & 28 & 73.7 \\
\hline \multicolumn{3}{|l|}{ Grade } \\
\hline Low & 26 & 68.4 \\
\hline High & 12 & 31.6 \\
\hline \multicolumn{3}{|l|}{ Necrosis } \\
\hline Yes & 10 & 26.3 \\
\hline No & 28 & 73.7 \\
\hline \multicolumn{3}{|l|}{ Recurrence } \\
\hline Yes & 10 & 26.3 \\
\hline No & 28 & 73.7 \\
\hline
\end{tabular}

SD, standard deviation; CRP, C-reactive protein; DM, diabetes mellitus; HT, hypertension; CAD, coronary artery disease; GPS, Glasgow prognostic score.

gender and $\mathrm{BMI}(\mathrm{P}>0.05)$. The mean tumor diameter of patients who developed recurrence was significantly higher compared with that in patients without recurrence $(\mathrm{P}=0.002)$. No statisti- cally significant difference was found between patients with and those without recurrence regarding the incidence of DM, HT and CAD, or the frequency of increased CRP levels ( $\mathrm{P}>0.05)$.

Hypoalbuminemia was significantly more frequent in cases with compared with those without recurrence (40 vs. $7.1 \%$, respectively; $\mathrm{P}=0.031$ ) and the risk of recurrence in cases with hypoalbuminemia was increased 8.67 -fold [odds ratio $(\mathrm{OR})=8.667$; 95\% confidence interval (CI): 1.276-58.849). GPS scores of 1 and 2 were significantly more frequently observed in cases with recurrence compared with those without recurrence ( 60 vs. $7.1 \%$, respectively; $\mathrm{P}=0.002$ ), whereas the risk of recurrence was 19.5-fold higher in patients with GPS scores of 1 and 2 (OR=19.500; 95\% CI: 2.872-132.410). T1 tumors were significantly more frequent among patients with recurrence compared with those without recurrence (60 vs. $14.3 \%$, respectively; $\mathrm{P}=0.011)$ and the risk of recurrence was 9-fold higher in patients with $\mathrm{T} 1$ tumors (OR=9.000; 95\% CI: 1.729-46.842). A significantly higher percentage of patients with recurrence had HG tumors compared with those without recurrence ( 80 vs. $14.3 \%$, respectively; $\mathrm{P}=0.001$ ) and an 24 -fold higher risk of recurrence was associated with $\mathrm{HG}$ tumors $(\mathrm{OR}=24.000$; 95 CI\%: 3.675-156.738). Tumor necrosis was observed in a significantly higher percentage of cases with recurrence compared with recurrence-free patients ( $80 \mathrm{vs.} 7.1 \%$, respectively; $\mathrm{P}=0.001$ ) and the risk of recurrence was increased 52-fold in cases with tumor necrosis $(\mathrm{OR}=52.000 ; 95 \mathrm{CI} \%$ : 6.279-430.668).

Association of clinicopathological parameters with tumor necrosis. Regarding the association of clinicopathological parameters with tumor necrosis, the mean tumor diameter in patients with necrosis was significantly higher compared with that in patients without necrosis $(\mathrm{P}=0.028)$. The mean albumin levels in cases exhibiting tumor necrosis were significantly lower compared with those in cases without necrosis $(\mathrm{P}=0.031)$ and the risk of tumor necrosis in cases with hypoalbuminemia was increased 8.67-fold (OR=8.667; 95\% CI: 1.276-58.849). In patients with tumor necrosis, GPS scores of 1 and 2 were significantly more frequent compared with patients without necrosis (60 vs. 7.1\%, respectively; $\mathrm{P}=0.002$ ) and the risk of tumor necrosis was increased 19.5-fold in cases with GPS scores of 1 and $2(\mathrm{OR}=19.500 ; 95 \% \mathrm{CI}: 2.872-132.410)$. In patients with tumor necrosis, T1 tumors were significantly more frequent compared with cases without necrosis (60 vs. $14.3 \%$, respectively; $\mathrm{P}=0.011$ ) and the risk of recurrence was increased 9-fold in cases with $\mathrm{T} 1$ tumors (OR=9.000; 95\% CI: 1.729-46.842). An increased frequency of $\mathrm{HG}$ tumors was encountered among cases with tumor necrosis compared with patients without any evidence of necrosis ( 80 vs. $14.3 \% ; \mathrm{P}=0.001$ ) and the risk of tumor necrosis was increased 24-fold in patients with $\mathrm{HG}$ tumors (OR=24.000; 95\% CI: 3.675-156.738).

Association of clinicopathological parameters with tumor grade. The association of clinicopathological parameters with tumor grade was also evaluated. The mean age of patients with HG tumors was significantly higher compared with that of patients with lower-grade tumors $(\mathrm{P}=0.035)$. However, $\mathrm{CRP}$ levels, hypoalbuminemia or GPS were not found to be significantly associated with tumor grade $(\mathrm{P}>0.05)$. T1 was more frequent among cases with HG tumors compared with patients with lower grades (66.7 vs. $7.7 \%$, respectively; $\mathrm{P}=0.001$ ). 
Table III. Evaluation of parameters affecting the recurrence of primary bladder cancer.

A, mean values of baseline parameters according to recurrence and non-recurrence

\begin{tabular}{lccc}
\hline Parameters & Recurrence $($ mean $\pm \mathrm{SD})(\mathrm{n}=10)$ & No recurrence $($ mean \pm SD) $(\mathrm{n}=28)$ & $\mathrm{P}-\mathrm{value} \mathrm{a}^{\mathrm{a}}$ \\
\hline Age $($ years $)$ & $63.0 \pm 6.25$ & $59.14 \pm 10.59$ & 0.287 \\
BMI $\left(\mathrm{kg} / \mathrm{m}^{2}\right)$ & $26.16 \pm 1.93$ & $26.11 \pm 2.48$ & 0.955 \\
Tumor diameter $(\mathrm{mm})$ & $41.10 \pm 16.56$ & $24.39 \pm 12.60$ & $0.002^{\mathrm{b}}$ \\
\hline
\end{tabular}

B, no. of patients according to recurrence and non- recurrence

\begin{tabular}{|c|c|c|c|}
\hline Parameters & Recurrence, n (\%) & No recurrence, n (\%) & P-value ${ }^{c}$ \\
\hline Gender & & & 0.279 \\
\hline Female & $2(20)$ & $2(7.1)$ & \\
\hline Male & $8(80)$ & $26(92.9)$ & \\
\hline $\mathrm{DM}$ & $0(0)$ & $6(21.4)$ & 0.168 \\
\hline $\mathrm{HT}(\mathrm{mm} \mathrm{Hg})$ & $2(20)$ & $12(42.9)$ & 0.268 \\
\hline CAD & $4(40)$ & $10(35.7)$ & 1.000 \\
\hline Elevated CRP (mg/dl) & $6(60)$ & $22(78.6)$ & 0.404 \\
\hline Hypoalbuminemia $(<3.5 \mathrm{~g} / \mathrm{dl})$ & $4(40)$ & $2(7.1)$ & $0.031^{\mathrm{b}}$ \\
\hline GPS & & & $0.002 b$ \\
\hline 0 & $4(40)$ & $26(92.9)$ & \\
\hline $1-2$ & $6(60)$ & $2(7.1)$ & \\
\hline Tumor stage & & & $0.011 b$ \\
\hline $\mathrm{T} 1$ & $6(60)$ & $4(14.3)$ & \\
\hline $\mathrm{Ta}$ & $4(40)$ & $24(85.7)$ & \\
\hline $\mathrm{HG}$ & $8(80)$ & $4(14.3)$ & $0.001^{\mathrm{d}}$ \\
\hline Necrosis & $8(80)$ & $2(7.1)$ & $0.001^{\mathrm{d}}$ \\
\hline
\end{tabular}

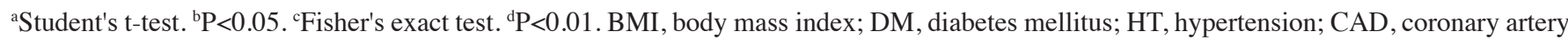
disease; CRP, C-reactive protein; GPS, Glasgow prognostic score; HG, high-grade; SD, standard deviation.

\section{Discussion}

Inflammation in the tumor microenvironment significantly contributes to the survival and proliferation of malignant cells and promotes tumor angiogenesis, invasion and metastasis (5). Inflammation affects numerous aspects of cancer, including tumor development, progression, clinical presentation and prognosis. Systemic inflammatory and immune responses to tumor cells and tumor cell-secreted peptides depend on the type and extent of the malignancy and may significantly affect outcome. However, current prognostic methods do not generally take inflammatory factors into consideration. Accumulating evidence indicates that SIR markers, including cytokines, albumin, CRP, white blood cell count and serum amyloid A, may serve as independent prognostic factors in cancer patients (6). The immune system has a dual function in the development as well as in the progression of cancer; it may destroy tumor cells, but it may also promote their growth and their ability for invasion and migration.

Over the last few years, a number of studies have assessed the association of local inflammatory infiltration in the tumor bed with patient survival (7-9). These studies demonstrated that lymphocyte subgroups of $\mathrm{CD}^{+}$and $\mathrm{CD} 4^{+} \mathrm{T}$ cells provide prognostic information in various tumor types, including colorectal cancer, renal cell carcinoma and TCC of the urinary bladder, with diverse degrees of infiltration $(8,9)$. However, as the evaluation of lymphocyte infiltration is time-consuming, it has not yet been introduced into routine clinical practice.

Studies performed over the last decade have demonstrated the prognostic value of SIR in the prediction of survival. Elevated CRP has been found to be an independent predictor of survival in patients with solid tumors, including lung, esophageal, kidney and prostate cancer, as well as BC (4,10-13).

The potential use of CRP as a biomarker in urological cancers has been extensively investigated and CRP was included among the prognostic criteria of the National Institutes of Health of the USA (14). CRP has been reported to be associated with poor prognosis and the presence of metastatic disease in patients with prostate cancer (15). Gakis et al (16) retrospectively analyzed patients who had undergone radical cystectomy and demonstrated that CRP in combination with other parameters provided additional prognostic information on muscle-invasive BC. 
CRP is a marker of acute-phase inflammation, which is cost-effective and non-invasive, whereas albumin is a negative acute-phase protein and its levels decrease as the concentration of CRP increases; it is currently used as an SIR marker rather than a nutritional status marker (17). Increased albumin catabolism, chronic malnutrition and chronic inflammatory reaction due to carcinogenesis may represent etiological factors for hypoalbuminemia. Furthermore, in association with interleukin-6, the use of albumin in the production of cytokines in hepatocytes may result in hypoalbuminemia (18). Another hypothesis is associated with increased microvascular penetration and transcapillary passage of albumin under the influence of tumor necrosis factor (19).

It has been demonstrated that hypoalbuminemia provides prognostic information in various types of cancer. Gregg et al (20) reported that preoperative albuminemia is a predictor of decreased survival. Furthermore, hypoalbuminemia was found to be an independent marker of worse prognosis in patients diagnosed with hepatocellular carcinoma (21). However, numerous studies have demonstrated that tumor recurrence developed during the early stages of colon and rectal cancer and that mortality following curative resection was associated with preoperative hypoalbuminemia.

GPS is an inflammation-based prognostic marker constructed upon evaluation of preoperative serum CRP and albumin levels (22). Proctor et al (23) reported that, as a powerful prognostic factor independent of tumor location, the modified GPS was superior to the classical GPS in cancer patients. This outcome was based on the observation that hypoalbuminemia is commonly accompanied by elevated CRP, and that hypoalbuminemia per se is not associated with worse survival (23). However, in the present study, hypoalbuminemia and classical GPS were found to be successful in predicting recurrence in TCC of the bladder. Therefore, the use of the classical rather than the modified GPS was considered to be more appropriate for this type of cancer.

In the present study, 10 of the 38 patients (26.3\%) with primary $\mathrm{BC}$ had elevated CRP levels prior to surgery, while CRP concentrations were within normal limits in the remaining 28 patients (73\%). However, intergroup comparisons of CRP levels did not reveal any statistically significant differences. Hypoalbuminemia was detected in $6(15.8 \%)$ of the 38 cases with primary $\mathrm{BC}$, while the albumin levels in the remaining 32 patients $(84.2 \%)$ were within the normal range. Of note, the incidence of hypoalbuminemia was significantly higher in cases with recurrence compared with that in patients without recurrence ( 40 vs. $7.1 \%$, respectively; $\mathrm{P}<0.05$ ). The recurrence rate was 8.67 -fold higher in cases with preoperative hypoalbuminemia. Furthermore, hypoalbuminemia was more frequent in patients with tumor necrosis compared with patients without necrosis. The risk of recurrence in patients who presented with tumor necrosis was increased 52-fold. This statistically significant association between hypoalbuminemia and tumor necrosis again confirms the role of hypoalbuminemia in the prediction of recurrence in patients with $\mathrm{BC}$.

A study performed by Al Murri et al (24) revealed that, among systemic inflammation markers other than CRP, hypoalbuminemia is an independent and significant predictor in survey studies on primary operable breast cancer, which is in line with the results of the present study.
Regarding the predictive value of the GPS scores, the recurrence risk was found to be increased 19.5 -fold in cases with GPS scores of 1 and 2 compared with those with GPS scores of 0 . In patients with HG tumors, the risk of recurrence was increased 24-fold compared with that in patients with LG tumors. In patients with $\mathrm{T} 1$ disease, the risk of recurrence was 9 times higher compared with that in patients with Ta disease. Tumor size was also associated with the risk of recurrence.

The prognostic role of the tumor necrosis factor in renal, breast, pulmonary and colorectal malignancies has been documented $(25,26)$. However, only a limited number of studies have assessed the prognostic role of tumor necrosis factor in BC. Based on the results of the present study, the risk of recurrence in cases with primary BC was increased 52-fold in patients with tumor necrosis.

The underlying mechanisms associated with the prognostic role of the SIR in the prediction of cancer-specific survival have not been fully elucidated. However, as a component of the SIR, the release of pro-inflammatory cytokines and growth factors into the systemic circulation has already been recognized. Furthermore, tumor growth factors are considered to promote cytokine release. SIR may be activated secondary to local tissue damage caused by tumor necrosis or tumor host cells (4).

There is accumulating evidence regarding the predictive value of the preoperative levels of serum SIR markers and GPS regarding patient prognosis and recurrence for the management of various tumor types. The aim of the present study was to investigate the value of preoperative serum albumin and CRP levels, as well as the GPS, in the prediction of recurrence and prognosis of BC. Serum albumin and CRP are easily available, well-standardized, time- and cost-effective biomarkers, and their measurement may be added to the routine preoperative laboratory tests. In particular, hypoalbuminemia and elevated GPS scores were shown to be of significant predictive value for the recurrence of bladder TCC, and may be used as an independent parameter in this type of BC.

\section{References}

1. American Cancer Society (ACS): Cancer Facts and Figures 2009. ACS, Atlanta, 2009.

2. Lucca I, de Martino M, Klatte T and Shariat SF: Novel biomarkers to predict response and prognosis in localized bladder cancer. Urol Clin North Am 42: 225-233, 2015.

3. Vakkila J and Lotze MT: Inflammation and necrosis promote tumor growth. Nat Rev Immunol 4: 641-648, 2004.

4. Scott HR, McMillan DC, Forrest LM, Brown DJ, McArdle CS and Milroy R: The systemic inflammatory response, weight loss, performance status and survival in patients with inoperable non-small cell lung cancer. Br J Cancer 87: 264-267, 2002.

5. Mantovani A, Allavena P, Sica A and Balkwill F: Cancer-related inflammation. Nature 454: 436-444, 2008.

6. Moore MM, Chua W, Charles KA and Clarke SJ: Inflammation and cancer: Causes and consequences. Clin Pharmacol Ther 87: 504-508, 2010.

7. Nielsen HJ, Hansen U, Christensen IJ, Reimert CM, Brünner N and Moesgaard F: Independent prognostic value of eosinophil and mast cell infiltration in colorectal cancer tissue. J Pathol 189: 487-495, 1999.

8. Nakano O, Sato M, Naito Y, Suzuki K, Orikasa S, Aizawa M, Suzuki Y, Shintaku I, Nagura H and Ohtani H: Proliferative activity of intratumoral $\mathrm{CD}^{+} \mathrm{T}$-lymphocytes as a prognostic factor in human renal cell carcinoma: Clinicopathologic demonstration of antitumor immunity. Cancer Res 61: 5132-5136, 2001. 
9. Hilmy M, Campbell R, Bartlett JM, McNicol AM, Underwood MA and McMillan DC: The relationship between the systemic inflammatory response, tumor proliferative activity, T-lymphocytic infiltration and COX-2 expression and survival in patients with transitional cell carcinoma of the urinary bladder. Br J Cancer 95: 1234-1238, 2006.

10. Roxburgh CS, Salmond JM, Horgan PG, Oien KA and McMillan DC: Comparison of the prognostic value of inflammation-based pathologic and biochemical criteria in patients undergoing potentially curative resection for colorectal cancer. Ann Surg 249: 788-793, 2009.

11. Crumley AB, McMillan DC, McKernan M, McDonald AC and Stuart RC: Evaluation of an inflammation-based prognostic score in patients with inoperable gastro-oesophageal cancer. Br J Cancer 94: 637-641, 2006.

12. Lamb GW, McArdle PA, Ramsey S, McNichol AM, Edwards J, Aitchison M and McMillan DC: The relationship between the local and systemic inflammatory responses and survival in patients undergoing resection for localized renal cancer. BJU Int 102: 756-761, 2008.

13. McArdle PA, Qayyum T and McMillan DC: Systemic inflammatory response and survival in patients with localized prostate cancer: 10-year follow-up. Urol Int 84: 430-435, 2010.

14. Saito K and Kihara K: Role of C-reactive protein in urological cancers: A useful biomarker for predicting outcomes. Int J Urol 20: 161-171, 2013.

15. McMillan DC: The systemic inflammation-based Glasgow prognostic score: A decade of experience in patients with cancer. Cancer Treat Rev 39: 534-540, 2013

16. Gakis G, Todenhöfer T, Renninger M, Schilling D, Sievert KD, Schwentner C and Stenzl A: Development of a new outcome prediction model in carcinoma invading the bladder based on preoperative serum C-reactive protein and standard pathological risk factors: The TNR-C score. BJU Int 108: 1800-1805, 2011.

17. McMillan DC: Systemic inflammation, nutritional status and survival in patients with cancer. Curr Opin Clin Nutr Metab Care 12: 223-226, 2009.
18. Heys SD, Walker LG, Deehan DJ and Eremin OE: Serum albumin: a prognostic indicator in patients with colorectal cancer. J R Coll Surg Edinb 43: 163-168, 1998.

19. $\mathrm{Ku} \mathrm{JH}$, Kim M, Choi WS, Kwak C and Kim HH: Preoperative serum albumin as a prognostic factor in patients with upper urinary tract urothelial carcinoma. Int Braz J Urol 40: 753-762, 2014.

20. Gregg JR, Cookson MS, Phillips S, Salem S, Chang SS, Clark PE, Davis R, Stimson CJ Jr, Aghazadeh M, Smith JA Jr and Barocas DA: Effect of preoperative nutritional deficiency on mortality after radical cystectomy for bladder cancer. J Urol 185: 90-96, 2011.

21. Cho YK, Chung JW, Kim JK, Ahn YS, Kim MY, Park YO, Kim WT and Byun JH: Comparison of 7 staging systems for patients with hepatocellular carcinoma undergoing transarterial chemoembolization. Cancer 112: 352-361, 2008

22. McMillan DC: An inflammation-based prognostic score and its role in the nutrition-based management of patients with cancer. Proc Nutr Soc 67: 257-262, 2008.

23. Proctor MJ, Morrison DS, Talwar D, Balmer SM, Fletcher CD, O'Reilly DS, Foulis AK, Horgan PG and McMillan DC: A comparison of inflammation-based prognostic scores in patients with cancer. A Glasgow Inflammation Outcome Study. Eur J Cancer 47: 2633-2641, 2011.

24. Al Murri AM, Doughty JC, Lannigan A, Wilson C, McArdle CS and McMillan DC: The relationship between deprivation, tumor stage and the systemic inflammatory response in patients with primary operable breast cancer. Br J Cancer 91: 1063-1065, 2004.

25. Pollheimer MJ, Kornprat P, Lindtner RA, Harbaum L, Schlemmer A, Rehak P and Langner C: Tumor necrosis is a new promising prognostic factor in colorectal cancer. Hum Pathol 41: 1749-1757, 2010.

26. Sengupta S, Lohse CM, Leibovich BC, Frank I, Thompson RH, Webster WS, Zincke H, Blute ML, Cheville JC and Kwon ED: Histologic coagulative tumor necrosis as a prognostic indicator of renal cell carcinoma aggressiveness. Cancer 104: 511-520, 2005. 